\title{
V. The Dynamical Behaviour of the Age-Size-Distribution of a Cell Population
}

\author{
H.J.A.M. Heijmans
}

\section{Introduction}

It is appropriate to think of the cell cycle as an ordered sequence of biochemical events, such as the synthesis of RNA and proteins and the replication of DNA, finally ending up in cell division. The rate at which these biochemical events, such as the increase of structural materials, proceed may heavily depend on volume (size) through such factors as diffusion times and surface to volume ratios. In section 1.4 and chapter II we therefore considered the case that the position of a particular cell in its cycle was adequately described by its size. However, some of the biochemical reactions seem to proceed sequentially during a cell's life cycle and for such reactions cell age provides a better description. In this chapter we therefore consider the Bell-Anderson model for cell growth and division, the main assumption being that the $i$-state of a cell is given by a vector $(a, x)$ where $a$ and $x$ represent age and size respectively. The $i$-state space $\Omega$ is a subset of $\mathbb{R}^{+} \times \mathbb{R}^{+}$. In our study we restrict ourselves to the case that the growth rate of individual cells only depends on their size: see section 1 . This study is certainly not the only one where age and size are considered to be the relevant parameters structuring microbial populations. Besides the paper by BELL \& ANDERSON (1967), where one can find a derivation of the model discussed here, we mention Sinko \& STreIFER (1967), Bell (1968), Trucco (1970), Trucco \& Bell (1970), Hannsgen, Tyson \& Watson (1985) and TYson \& Hannsgen (1985b,) 198?, 1985a) for some related work in this area (see also section 9). Actually Sinko \& Streifer independently derived the model discussed here, and they applied it to populations of the pianarian worm Dugesia tigrina. In all the other papers variants of the model are investigated mathematically. In all of these papers, except the two last ones, one of the main restrictions is that the cell division probability only depends on age: TYSON and HANNSGEN (1985a) consider the so-called "tandem model" which is the transition probability model supplied with a critical size requirement. For a recent overview of some of this literature we refer to two articles written by JOHN TYsON (1985a,b).

In this chapter the division probability is allowed to depend on both age and size. After making some assumptions on the death, division, and growth rates we prove existence and uniqueness of solutions, after reducing the problem, by integration along the characteristics, to an abstract renewal equation for the birth function $B(t, x)$; see sections 2 and 3. Here $x$ is "size at birth", and the word "abstract" means that the birth function is not just a scalar function of time, as it was in chapter IV, but takes its values in a Banach space; c.f. chapter II.

To determine the asymptotic behaviour of solutions, we first apply Laplace transformation to the renewal equation. This is done in section 4. Subsequently we write down the characteristic equation (which now, of course, is not of scalar type, but involves Banach space operators) and prove the existence of a dominant root, i.e. a root with largest real part (see section 6 for the details). This requires a certain amount of spectral theory. (In section 5 we give some results from the theory of positive operators, which are used in section 6.) It turns out that we need an extra condition on the growth rate to carry out this program, viz. assumption 6.4 , which resembles the one made in chapter II. In the sections 7 and 8 we compute the residue associated with this dominant root, and apply the inverse Laplace transformation, which gives us the asymptotic behaviour of solutions. In section 9 it is argued that we cannot dispense with the extra condition on the growth rate. This disproves the supposition of BeLl (1968) that, even in the case of exponential individual growth, a stable age-size distribution might exist if the division probability depends in an appropriate manner on age and size. BeLL (1968) argued (and this indeed is correct) that this is impossible if the division probability depends on age only, and individual cells grow exponentially in size; a rigorous proof of this result is given in TRUCCO \& BELI (1970), where the first and second moments of the distribution of birth sizes are computed.

The approach we have adopted in this chapter (i.e. reduction to a renewal equation) is not as different from the semigroup approach of chapter II as it seems at first sight; compare also section IV.2.3.

Finally we note that a reduction of the partial differential equation to a renewal equation for the birth function is generally possible. What one has to do is to replace the actual $i$-state vector $\underline{x}$ by the vector representing 
chronological age $a$ plus state at birth $x_{b}$ : note that this new vector is one dimension higher if $a$ was not already contained in $x$. Then, by integration along the characteristics, one finds a renewal equation for $B\left(t, x_{b}\right)$, representing the rate at which individuals with state $x_{b}$ are born at time $t$.

\section{The model}

We assume that a cell is fully characterized by its age a and size x. Here size can mean volume, length, DNA-content or any other quantity which obeys a physical conservation law. Size increases with time and we assume that this process can be described by the ordinary differential equation

$$
\frac{d x}{d t}=g(x) \text {. }
$$

This means in particular that the growth rate $\mathrm{g}$ does neither depend on age, which seems very reasonable from a biological point of view, nor on environmental factors (such as food density) which are influenced by the population itself, causing nonlinearities in the equation. Age also increases with time and obeys $\frac{d a}{d t}=1$. However our theory can be easily extended to the case where $a$ denotes some physiological age, which does not necessarily increase linearly with time: $\frac{d a}{d t}=f(a)$ where $f$ is a bounded continuous positive function. We assume that if a cell divides, it produces two daughter cells, both having age zero and half the size of the mother. Let $n(t, a, x)$ be the cell density function, i.e. $\int_{x_{1}}^{x_{2}} \int_{a_{1}}^{a_{2}} n(t, a, x) d a d x$ is the number of cells having age between $a_{1}$ and $a_{2}$, and size between $x_{1}$ and $x_{2}$. From the conservation principle it follows that the equation for the density function can be written as

$$
\frac{\partial n}{\partial t}=-\nabla \cdot J-F-D
$$

where the flux $J=J(t, a, x)$ is given by $J=(n(t, a, x), g(x) n(t, a, x))^{T}$, and $\nabla$ is the operator $\left(\frac{\partial}{\partial a}, \frac{\partial}{\partial x}\right)^{T}$. The sinks $F$ and $D$ account for the individuals which "disappear" as a result of fission and death respectively. We refer to chapter III for a more general description how to derive balance equations such as (1.2).

Let fission and death be described by the per capita probabilities per unit of time $b(a, x)$ and $\mu(a, x)$ respectively, then $F=F(t, a, x)=b(a, x) n(t, a, x)$ and $D=D(t, a, x)=\mu(a, x) n(t, a, x)$.

We shall now introduce a number of mathematical assumptions on the functions $g, b$ and $\mu$ and discuss their biological meaning and/or mathematical motivation. With respect to the growth rate $g$ we assume

$$
\begin{aligned}
& \left(A_{g}\right) \quad g \text { is a continuous function on }[0, \infty) \text { and there exist constants } g_{\min }, g_{\max } \\
& \text { such that } 0<g_{\min } \leqslant g_{\max }<\infty \text { and } g_{\min } \leqslant g(x) \leqslant g_{\max } \text { for all } x \in[0, \infty) .
\end{aligned}
$$

It follows from this assumption that certain combinations of $a$ and $x$ are forbidden in the sense that cells with such a combination of age and size will never come into existence. More precisely there exists a (continuous) curve in the $(a, x)$-plane starting from $(a, x)=(0,0)$ and tending towards $(\infty, \infty)$ below which no individual will ever dwell.

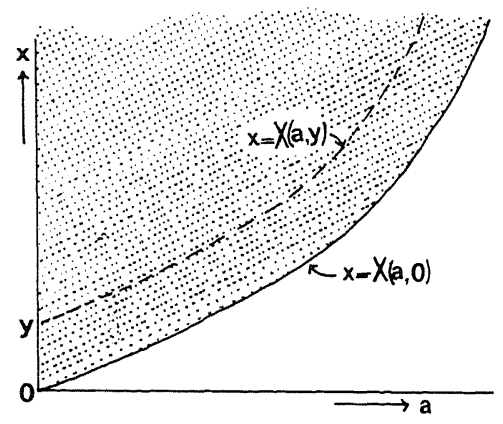

Figure 1.1. The set $\Omega$. An individual with birth size $y$ travels along the curve $\{X(a, y) \mid a \geqslant 0\}$ until it dies or divides.

We can compute this curve explicitly. Consider a cell whose size at birth is $x \geqslant 0$ (assuming that such cells indeed exist). Let $X(a, x)$ be its size at age $a$, if it has not died or divided before reaching that age. Then $X$ is the solution of 
the initial value problem $\frac{d X}{d a}=g(X), X(0)=x$, which has a continuous (differentiable) solution tending to $\infty$ if $a$ tends to $\infty$ because of assumption $\left(A_{g}\right)$. The curve $\{(a, X(a, y)) \mid a \geqslant 0\}$ is called the characteristic curve starting from $(0, y)$ (see figure 1.1). We refer to section 2 for more details.

Individuals can only exist in the shaded region $\Omega=\left\{(a, x) \in \mathbb{R}^{+} \times \mathbb{R}^{+} \mid x \geqslant X(a, 0)\right\}$. The actual state space $\Omega_{s}$ (i.e. the subset of $\mathbb{R}^{+} \times \mathbb{R}^{+}$in which indeed individuals do occur) is a subset of $\Omega$, and in some cases $\Omega_{s}$ is smaller than $\Omega$. (We refer to section 6 for an example.)

We impose the following conditions on $b$ and $\mu$ :

$$
\begin{aligned}
\left(A_{b}\right) \quad & \left.b \in L_{\infty}(\Omega) \text { (i.e. } b \text { is measurable and essentially bounded on } \Omega\right) \\
& b(a, x)=0, a \leqslant a_{0},(a, x) \in \Omega, \\
& b(a, x)>0, a>a_{0},(a, x) \in \Omega, \\
& \liminf _{a \rightarrow \infty} b(a, X(a, x)) \geqslant \underline{b}>0 \text { for every } x .
\end{aligned}
$$

Here $a_{0}>0$ is some threshold below which cells cannot divide. The biological reason for this is that every cell has to go through a phase during which DNA is replicated, and the duration of this phase is more or less constant (see BELL \& ANDERSON (1967), EISEN (1979)). Biologically, the last condition in $\left(A_{b}\right)$ means that old individuals continue dividing at a positive rate.

$$
\begin{gathered}
\left(A_{\mu}\right) \quad \mu \in L_{\infty}^{l o c}(\Omega), \text { i.e. } \mu \text { is measurable and essentially bounded on compact subsets of } \Omega, \\
\mu(a, x) \geqslant 0,(a, x) \in \Omega .
\end{gathered}
$$

Let

$$
d(a, x)=b(a, x)+\mu(a, x) .
$$

We assume

$\left(A_{d}\right) \quad$ There exists a constant $d_{\infty}$ with $0<d_{\infty} \leqslant \infty$ such that $\lim _{\sigma \rightarrow \infty} d(a+\sigma, X(\sigma, x))=d_{\infty}$ uniformly in $a$ and $x$. Moreover, if $d_{\infty}<\infty$, there exists a constant $M \geqslant 0$ such that for all $a$ and $x: \int_{0}^{\infty}\left|d(a+\sigma, X(\sigma, x))-d_{\infty}\right| d \sigma \leqslant M$.

Biologically assumption $\left(A_{d}\right)$ means that the probability for a cell to reach age $a$ without dying or dividing decreases more or less exponentially if $a$ becomes large. In section 9 it is explained why this assumption is needed.

We can rewrite (1.2) as

$$
\begin{aligned}
& \frac{\partial}{\partial t} n(t, a, x)+\frac{\partial}{\partial a} n(t, a, x)+\frac{\partial}{\partial x}(g(x) n(t, a, x))=-(\mu(a, x)+b(a, x)) n(t, a, x), \\
& t \geqslant 0,(a, x) \in \Omega .
\end{aligned}
$$

The fact that dividing mothers of age $a$ and size $2 x$ give birth to two daughters of age 0 and size $x$ is accounted for by the boundary condition

$$
n(t, 0, x)=4 \int_{a_{0}}^{\infty} b(a, 2 x) n(t, a, 2 x) d a .
$$

See BELL \& ANDERSON (1967) or chapter I for an explanation of the factor 4.

REMARK 1.1. In (1.5) we only have to integrate over those ages $a$ that satisfy $X(a, 0) \leqslant 2 x$.

We specify an initial condition

$$
n(0, a, x)=n_{0}(a, x),(a, x) \in \Omega .
$$

Biological considerations yield that $n_{0}$ should satisfy

$$
n_{0}(a, x) \geqslant 0,(a, x) \in \Omega \text { and } n_{0} \in L_{1}(\Omega) .
$$




\section{Reduction to an abstract renewal equation}

Usually age-dependent population models are reduced to a renewal equation (which is a Volterra integral equation of convolution type) for the birth function (see chapter IV)). Here we will show that this can also be done for our agesize-structured model (1.4)-(1.6). In this case, however, we obtain an abstract renewal equation, in the sense that solutions take values in some function space.

Let $m(t, a, x)$ be defined by

$$
m(t, a, x)=g(x) n(t, a, x),
$$

then $m$ satisfies the equation

$$
\begin{aligned}
& \frac{\partial m}{\partial t}+\frac{\partial m}{\partial a}+g(x) \frac{\partial m}{\partial x}=-(\mu(a, x)+b(a, x)) m(t, a, x), \\
& m(t, 0, x)=\frac{4 g(x)}{g(2 x)} \int_{a_{0}}^{\infty} b(a, 2 x) m(t, a, 2 x) d a, \\
& m(0, a, x)=m_{0}(a, x) \stackrel{\text { def }}{=} g(x) n_{0}(a, x) .
\end{aligned}
$$

By the method of integration along characteristics (see Courant \& Hilbert (1962)) we can convert this system into an integral equation.

The characteristic curve through $(t, a, x)$ is determined by $s \rightarrow(T(s, t), A(s, a), X(s, x))$, where $s$ is an independent book-keeping variable and $T, A, X$ are solutions of the ODE's $\frac{d T}{d s}=1, T(0, t)=t, \frac{d A}{d s}=1, A(0, a)=a$, $\frac{d X}{d s}=g(X), X(0, x)=x$, thus $T(s, t)=s+t, A(s, a)=s+a$, and $X(s, x)=G^{-1}(s+G(x))$, where

$$
G(x)=\int_{0}^{x} \frac{d \xi}{g(\xi)}, x \geqslant 0,
$$

can be interpreted as the time needed to grow from 0 to $x$ and $G^{-1}$ denotes its inverse. Observe that $G^{-1}(a)=X(a, 0)$.

Now let $t, a, x$ be fixed and let $\bar{m}(s)=m(T(s, t), A(s, a), X(s, x))$, then

$$
\frac{d \bar{m}}{d s}=-d(A(s, a), X(s, x)) \bar{m}(s),
$$

where $d(a, x)$ is given by (1.3). Let

$$
Q(s, a, x) \stackrel{\text { def }}{=} \exp \left[-\int_{0}^{s} d(A(\sigma, a), X(\sigma, x)) d \sigma\right],
$$

which can be interpreted as the probability that a cell with age $a$ and size $x$ reaches age $a+s$. From (2.4) we obtain that

$$
\bar{m}(s)=\bar{m}(0) Q(s, a, x)
$$

Let

$$
t^{\prime}=T(s, t), a^{\prime}=A(s, a), x^{\prime}=X(s, x) .
$$

(i) We choose $t=0$. Then $a=a^{\prime}-t^{\prime}, x=X\left(-t^{\prime}, x^{\prime}\right)$. If we substitute this in (2.6) we obtain

$$
m\left(t^{\prime}, a^{\prime}, x^{\prime}\right)=m\left(0, a^{\prime}-t^{\prime}, X\left(-t^{\prime}, x^{\prime}\right)\right) \cdot Q\left(t^{\prime}, a^{\prime}-t^{\prime}, X\left(-t^{\prime}, x^{\prime}\right)\right) \text {, if } a^{\prime}>t^{\prime} .
$$

(ii) We choose $a=0$. Then $t=t^{\prime}-a^{\prime}, x=X\left(-a^{\prime}, x^{\prime}\right)$, and we deduce from (2.6)

$$
m\left(t^{\prime}, a^{\prime}, x^{\prime}\right)=m\left(t^{\prime}-a^{\prime}, 0, X\left(-a^{\prime}, x^{\prime}\right)\right) \cdot E\left(a^{\prime}, X\left(-a^{\prime}, x^{\prime}\right)\right), \text { if } a^{\prime}<t^{\prime},
$$

where

$$
E(a, x) \stackrel{\text { def }}{=} Q(a, 0, x)=\exp \left[-\int_{0}^{a} d(\sigma, X(\sigma, x)) d \sigma\right]
$$

is the probability that a cell having size $x$ at birth reaches age $a$.

If we drop the accents in (2.9) and (2.10), and use (2.1) and (2.2c) we find 


$$
\begin{aligned}
& n(t, a, x)=\frac{g(X(-t, x))}{g(x)} n_{0}(a-t, X(-t, x)) \cdot Q(t, a-t, X(-t, x)), \quad t<a, \\
& n(t, a, x)=\frac{g(X(-a, x))}{g(x)} n(t-a, 0, X(-a, x)) \cdot E(a, X(-a, x)), \quad t>a .
\end{aligned}
$$

Let the birth function $B$ be defined by

$$
B(t, x)=n(t, 0, x) .
$$

If we substitute (2.11)-(2.12) into (1.5), then we obtain the following integral equation for $B$ :

$$
B(t, x)=\Phi(t, x)+\int_{a_{0}}^{t} k(a, 2 x) B(t-a, X(-a, 2 x)) d a,
$$

where

$$
\Phi(t, x)=\frac{4 g(X(-t, 2 x))}{g(2 x)} \int_{t}^{\infty} b(a, 2 x) Q(t, a-t, X(-t, 2 x)) \cdot n_{0}(a-t, X(-t, 2 x)) d a,
$$

and

$$
k(a, x)=\frac{4 g(X(-a, x))}{g(x)} b(a, x) E(a, X(-a, x)) .
$$

$\Phi(t, x)$ is only defined for values of $x$ satisfying $G(2 x) \geqslant t$, and one should read $\Phi(t, x)=0$ if $G(2 x) \leqslant t$. Furthermore $k(a, x)=0$ if $a \leqslant a_{0}$ or $a \geqslant G(x)$, and $k(a, x) \geqslant 0$ if $a_{0} \leqslant a \leqslant G(x)$.

The integral equation (2.14) was also found by BELL (1968) but he only solved it for the special case that all cells divide at the same age (see also BEYER (1970)).

It follows from (2.11)-(2.12) that knowledge of the solution $B(t, x)$ of $(2.14)$ yields the solution $n(t, a, x)$ of $(1.4)-$ (1.6). Therefore we shall concentrate on (2.14) during the rest of this chapter. In section 9 we shall interprete some result in terms of the density $n(t, a, x)$.

We can rewrite (2.14) as the abstract renewal equation

$$
B(t)=\Phi(t)+\int_{0}^{t} K(a) B(t-a) d a,
$$

where, for fixed $t \geqslant 0, \Phi(t) \in L_{1}[0, \infty)$ and $K(t)$ defines a bounded operator from $L_{1}[0, \infty)$ into itself:

$$
(K(t) \psi)(x)=k(t, 2 x) \psi(X(-t, 2 x)), \psi \in L_{1}[0, \infty),
$$

where one should read $\psi(X(-t, 2 x))=0$ if $G(2 x)<t$.

REMARK 2.1. Throughout this chapter we call a Banach space-valued function integrable if it is Bochner-integrable. This means the following: let $E$ be a Banach space with norm $\|\cdot\|_{E}$ and let $f:(a, b) \rightarrow E$, where $-\infty \leqslant a<b \leqslant \infty$. Then $f(t)$ is Bochner-integrable if and only if $f$ is strongly measurable and $\|f(t)\|_{E}$ is Lebesgue integrable (see HILLE \& Phillips (1957)).

$$
\begin{aligned}
& \text { We call } B(t) \text { a solution of }(2.17) \text { if and only if } \\
& \text { i) } B(t) \in L_{1}[0, \infty), t \geqslant 0, \\
& \text { ii) } \quad B(\cdot) \text { is integrable on }\left[0, t_{0}\right] \text { for all } t_{0} \geqslant 0, \\
& \text { iii) } \quad B(t) \text { obeys }(2.17) .
\end{aligned}
$$

\section{Existence and Uniqueness of solutions}

It turns out that the proof of an existence and uniqueness result for the abstract renewal equation (2.17) is rather similar to the scalar case which has been extensively treated in the book of BELLMAN \& COOKE (1963). First we shall prove a lemma.

LEMMA 3.1. (a) Let $d_{\infty}$ (of assumption $\left(A_{d}\right)$ ) be finite. Then there exist positive constants $T_{0}, m_{K}, M_{K}$ and $M_{\Phi}$ such that for all $t \geqslant T_{0}:\|\Phi(t)\| \leqslant M_{\Phi} e^{-d_{\infty} t}$, and for all $\psi \in L_{1}[0, \infty): m_{K} e^{-d_{\infty} t}\|\psi\| \leqslant\|K(t) \psi\| \leqslant M_{K} e^{-d_{\infty} t}\|\psi\|$.

(b) Let $d_{\infty}=\infty$. For all $c>0$ there exist constants $L_{K}(c), L_{\Phi}(c)>0$ such that for all $t \geqslant 0:\|\Phi(t)\| \leqslant L_{\Phi}(c) e^{-c t}$, $\|K(t) \psi\| \leqslant L_{K}(c) e^{-c t}\|\psi\|$, for all $\psi \in L_{1}[0, \infty)$. 
PROof. We shall only prove the second estimate in (a).

$$
E(a, x)=\exp \left[-\int_{0}^{a} d(\sigma, X(\sigma, x)) d \sigma\right]=\exp \left[-\int_{0}^{a}\left\{d(\sigma, X(\sigma, x))-d_{\infty}\right\} d \sigma\right] \cdot \exp \left[-\int_{0}^{a} d_{\infty} d \sigma\right] .
$$

Let $M$ be the constant of assumption $\left(A_{d}\right)$, then

$$
e^{-M} e^{-d_{\infty} a} \leqslant E(a, x) \leqslant e^{M} e^{-d_{\infty} a}
$$

The second part of (a) now follows immediately from these estimates and the assumptions $\left(A_{g}\right)$ and $\left(A_{b}\right)$. In an analogous manner we can prove part (b).

The following existence and uniqueness result can be proved.

THEOREM 3.2. Let $t_{0}>0$. There exists a unique bounded integrable solution $B(t)$ of $(2.17)$ on $\left[0, t_{0}\right]$.

The existence result can be established by the method of successive approximations. Uniqueness then follows from a Gronwall-type lemma. We refer to BeLlman \& COOKe (1963) where the scalar case has been worked out in great detail, and the reader will have no difficulty to see that all proofs can be carried over. Because $t_{0}$ can be chosen arbitrarily large, theorem 3.2 implies global existence of the solution $B(t)$.

REMARK 3.3. Strictly speaking condition $\left(A_{b}\right)$ and $\left(A_{\mu}\right)$ are sufficient to prove local existence and uniqueness.

In the next section we shall apply Laplace transformation to the integral equation (2.17). Therefore we need the following estimate.

THEOREM 3.4. There exists a $\beta \in \mathbb{R}$ such that $\|B(t)\| \leqslant M_{B} e^{\beta t}, t \geqslant 0$, where $M_{B}>0$ is a constant.

PROOF. Let $\beta \in \mathbb{R}$ be such that $\|\Phi(t)\| \leqslant c_{1} e^{\beta t}$ and $\int_{0}^{\infty} e^{-\beta t}\|K(t)\| d t=c_{2}<1$. From lemma 3.1 it is clear that such a $\beta$ indeed exists. Then

$$
\|B(t)\| \leqslant c_{1} e^{\beta t}+\int_{0}^{t}\|K(a)\| \cdot\|B(t-a)\| d a=c_{1} e^{\beta t}+e^{\beta t} \int_{0}^{t}\left\{\|K(a)\| \cdot e^{-\beta a}\right\} \cdot\left\{\|B(t-a)\| \cdot e^{-\beta(t-a)}\right\} d a .
$$

Let $v(t) \stackrel{\text { def }}{=} \max _{0 \leqslant a \leqslant t}\left\|B(a) e^{-\beta a}\right\|$, then $v(t) \leqslant c_{1}+v(t) \int_{0}^{t} e^{-\beta a}\|K(a)\| d a \leqslant c_{1}+c_{2} v(t)$, hence $v(t) \leqslant \frac{c_{1}}{1-c_{2}}$, from which we obtain that $\|B(t)\| \leqslant \frac{c_{1}}{1-c_{2}} e^{\beta t}$.

\section{Laplace Transformation}

A technique which turned out to be extremely useful in the study of scalar renewal equations is Laplace transformation (e.g. Bellman \& Cooke (1963), Hoppensteadt (1975) and chapter IV). This technique can also be employed in the study of abstract renewal equations such as (2.17). First we shall introduce some notations. Let $I \subseteq \mathbb{R}$ be an interval, and $E$ a Banach space. We define by $L_{p}(I, E), 1 \leqslant p \leqslant \infty$, the Banach space consisting of all functions $f: I \rightarrow E$ satisfying $\|f\|_{p} \stackrel{\text { def }}{=}\left\{\int_{l}\|f(t)\|^{p} d t\right\}^{\frac{1}{p}}<\infty$, if $p<\infty$ and $\|f\|_{\infty} \stackrel{\text { def }}{=}$ ess $\sup \|f(t)\|<\infty$, if $p=\infty$. If $I=[0, \infty)$ we shall write $L_{p}(0, \infty ; E)$ instead of $L_{p}([0, \infty) ; E)$.

REMARK 4.1. We have to distinguish between the norm of $f(t), t \geqslant 0$, as an element of $E$ and the norm of $f$ being an element of $L_{p}(I ; E)$. In the first case we write $\|f(t)\|$, in the second case $\|f\|_{p}$.

Definimion. Let $f$ be a function from $[0, \infty)$ to some Banach space $E$, then its Laplace transform $\hat{f}$ is defined by $\hat{f}(\lambda)=\int_{0}^{\infty} e^{-\lambda t} f(t) d t$, whenever this integral is defined with respect to the norm topology.

The following result is standard (HILle \& PHildips (1957)).

LEMMA 4.2. If $f \in L_{1}(0, \infty ; E)$ then $\hat{f}(\lambda)$ is analytic in $\operatorname{Re} \lambda>0$ and continuous in $\operatorname{Re} \lambda \geqslant 0$ (with respect to the norm- 
topology).

In what follows we need the Riemann-Lebesgue lemma (Hille \& PHillips (1957), thm 6.4.2). LEMMA 4.3 (Riemann-Lebesgue). Let $f \in L_{1}(0, \infty ; E)$ and $\hat{f}$ its Laplace transform. Then $\lim _{|\eta| \rightarrow \infty} \hat{f}(\xi+i \eta)=0$, uniformly
for $\xi$ in bounded closed subintervals of $(0, \infty)$.

Let the right-half-plane $\Lambda$ be defined by

$$
\Lambda \stackrel{\text { def }}{=}\left\{\lambda \in \mathbb{C} \mid \operatorname{Re} \lambda>-d_{\infty}\right\}
$$

(where $\Lambda=\mathbb{C}$ if $d_{\infty}=\infty$ ). Then it follows from lemma 3.1 and lemma 4.2 that $\hat{K}(\lambda)$ and $\hat{\Phi}(\lambda)$ are defined and analytic in $\Lambda$. Moreover it follows from lemma 3.1 that $\hat{K}(\lambda)$ is not defined if $\operatorname{Re} \lambda<-d_{\infty}$.

REMARK 4.4. It is not a priori clear whether $\hat{K}(\lambda)$ is defined for $\lambda$ on the vertical line $\operatorname{Re} \lambda=-d_{\infty}$. As to $\hat{\Phi}(\lambda)$ it depends on the initial age-size distribution $n_{0}(a, x)$ whether or not it is defined for values of $\lambda$ satisfying $\operatorname{Re} \lambda \leqslant-d_{\infty}$. However this is not important for our purposes.

We define $\hat{B}(\lambda)=\int_{0}^{\infty} e^{-\lambda t} B(t) d t$ for those values of $\lambda$ for which the integral converges. From theorem 3.4 we conclude that $\hat{B}(\lambda)$ exists if $\operatorname{Re} \lambda>\beta$. The convolution in (2.17) is converted by the Laplace transformation into a product of Laplace transforms. We wish to extend $\hat{B}(\lambda)$ to $\Lambda$ minus some set $\Sigma$ of singular points. More precisely

$$
\hat{B}(\lambda)=\hat{\Phi}(\lambda)+\hat{K}(\lambda) \hat{B}(\lambda), \lambda \in \Lambda \text {. }
$$

Let $\Sigma$ be the set of all $\lambda \in \Lambda$ for which $I-\hat{K}(\lambda)$ is singular.

$$
\Sigma=\{\lambda \in \Lambda \mid 1 \in \sigma(\hat{K}(\lambda)\},
$$

where $\sigma(\hat{K}(\lambda))$ denotes the spectrum of the operator $\hat{K}(\lambda)$. The condition $1 \in \sigma(\hat{K}(\lambda))$ is the usual precursor of a characteristic equation (HeImans (to appear), HoppensteadT (1975)).

For $\lambda \in \Lambda \backslash \Sigma$ we have

$$
\hat{B}(\lambda)=(I-\hat{K}(\lambda))^{-1} \hat{\Phi}(\lambda) \text {. }
$$

In section 8 we shall prove that the element $\lambda_{d}$ of $\Sigma$ with largest real part determines the large time behaviour of the solution $B(t)$. Often $\lambda_{d}$ turns out to be real, and the corresponding eigenvector of $\hat{K}\left(\lambda_{d}\right)$ to be positive: c.f. chapter II. The theory of positive operators is an important instrument to prove existence of $\lambda_{d}$, and has been succesfully exploited in a number of problems from population dynamics (Diekmann et al. (1984), Heumans (1984a), Heidmans (1985b)). As an intermezzo we shall now present some results from positive operator theory with the emphasis on the existence and uniqueness of positive eigenvectors and eigenfunctionals.

\section{Positive Operators}

For the basic theory of order structures in a Banach space and positive operators, we refer to SCHAEFER (1974).

In the sequel $E$ is some Banach space and $E^{*}$ is it's dual, i.e. the space of all linear functionals (or linear forms) on $E$. We denote the duality pairing of $\psi \in E, F \in E^{*}$ with $\langle F, \psi\rangle$. A subset $E+\subseteq E$ is called a cone if the following conditions are satisfied

(i) $E_{+}$is closed,

(ii) $\quad \alpha \phi+\beta \psi \in E_{+}$if $\phi, \psi \in E_{+}$and $\alpha, \beta \geqslant 0$

(iii) $\psi \in E_{+}$and $-\psi \in E_{+}$implies that $\psi=0$.

The reader can easily verify that by virtue of " $\phi \leqslant \psi$ iff $\psi-\phi \in E_{+}$" each cone $E_{+} \subseteq E$ defines an order relation on $E$ by which $E$ becomes an ordered Banach space. We say that $\phi<\psi$ if $\phi \leqslant \psi$ and $\phi \neq \psi$. The cone $E_{+}$is called total if the set $\left\{\psi-\phi \mid \psi, \phi \in E_{+}\right\}$is dense in $E$. The dual set $E_{+}^{*}$ is by definition the subset of $E^{*}$ consisting of all positive functionals on $E$, i.e. $F \in E_{+}^{*}$ if and only if $F \in E^{*}$ and $\langle F, \psi\rangle \geqslant 0$ for all $\psi \in E_{+}$. If $E_{+}$is total then $E_{+}^{*}$ is a cone as well. A positive functional $F$ is said to be strictly positive if $\langle F, \psi\rangle>0$ for all $\psi \in E_{+}, \psi \neq 0$. A bounded linear operator $T: E \rightarrow E$ is called positive (with respect to the cone $E_{+}$) if $T \psi \in E_{+}$for all $\psi \in E_{+}$. Notation $T \geqslant 0$. We denote the spectral radius of $T$ by $r(T)$. 
The first authors who systematically studied positive operators and their spectral properties were KREIN and RUTMAN (1948). In that paper they generalized the Frobenius theorem (which states that the spectral radius of a nonnegative matrix is an eigenvalue of that matrix). They proved, among others, the following result.

THEOREM 5.1 (KREIN \& RUTMAN (1948)). Let $T: E \rightarrow E$ be compact and positive with respect to the total cone $E_{+} \subseteq E$, and let $r=r(T)>0$. Then there exists $a \psi \in E_{+}, \psi \neq 0$ such that $T \psi=r \psi$.

They also introduced the notion of strong positivity. A positive operator $T: E \rightarrow E$ is called strongly positive if for all $\psi \in E_{+}, \psi \neq 0$ there is a natural number $p$ such that $T^{p} \psi \in E_{+}$, where $\dot{E}_{+}$denotes the interior of the cone $E_{+}$ (assuming that $E_{+}$has interior points). They proved that, if the assumptions of theorem 5.1 are fulfilled and, moreover, $T$ is strongly positive, then

(a) $\quad T$ has (except for a constant) one and only one eigenvector $\psi \in E_{+}$. Moreover $\psi \in E_{+}$and $T \psi=r \psi$.

(b) $T^{*}$ has one and only one eigenvector $F \in E_{+}^{*}, F$ is strictly positive and $T^{*} F=r F$.

(c) All other eigenvalues $\lambda$ of $T$ satisfy $|\lambda|<r(T)$.

Many years later their study was continued by a great number of authors, extending these ideas in several directions. Among others they weakend the condition that $T$ has to be compact; in many cases it is sufficient that $\lambda=r(T)$ is a pole of the resolvent $R(\lambda, T)=(\lambda I-T)^{-1}$. Furthermore several different concepts generalizing the concept of strong positivity have been introduced. We mention three of these generalizations. SCHAEFER (1974) introduced in the early sixties the concept of irreducible positive operators. KRASNOSELSKII (1964) studied $u_{0}$-positive operators, and finally SawASHIma (1964) developed the theory of non-supporting operators. (Sawashima uses the terminology "nonsupport".) All three concepts have the advantage that the interior of the cone $E_{+}$may be empty. The definitions of Schaefer and Sawashima are closely connected.

Defintrion (Sawashima (1964)). A bounded, positive operator $T: E \rightarrow E$ is called non-supporting with respect to $E_{+}$if for all $\psi \in E_{+}, \psi \neq 0$, and $F \in F_{+}^{*}, F \neq 0$, there exists an integer $p$ such that for all $n \geqslant p$ we have $\left\langle F, T^{n} \psi>>0\right.$.

The following result, which was proved by SAWASHIMA (1964) is needed in the following section. The result can also be found in paper by MAREK (1970) which provides a comprehensive overview of some of the developments in positive operator theory between 1950 and 1970.

THEOREM 5.2. Let the cone $E_{+}$be total, let $T: E \rightarrow E$ be non-supporting with respect to $E_{+}$, and suppose that $r=r(T)$ is a pole of the resolvent, then

(a) $\quad r>0$ and $r$ is an algebraically simple eigenvalue of $T$.

(b) The corresponding eigenvector $\psi$ satisfies: $\psi \in E_{+}$and $\langle H, \psi\rangle>0$ for all $H \in E_{+}^{*}, H \neq 0$.

(c) The corresponding dual eigenvector is strictly positive.

(d) If, in addition, $X$ is a Banach lattice and $\{\lambda \in \sigma(T):|\lambda|=r\}$ consists only of poles of the resolvent, then all remaining elements $\lambda \in \sigma(T)$ satisfy $|\lambda|<r$.

\section{Location of the singular points}

From now on we let $X=L_{1}[0, \infty)$. In section 4 we defined the analytic operator family $\hat{K}(\lambda), \lambda \in \Lambda$, being the Laplace transform of $K(t)$. Evidently $\hat{K}(\lambda)$ defines a bounded operator on $X$ for all $\lambda \in \Lambda$.

$$
\hat{K}(\lambda) \psi)(x)=\int_{a_{0}}^{G(2 x)} e^{-\lambda a} k(a, 2 x) \psi(X(-a, 2 x)) d a, \quad \psi \in X .
$$

In the Appendix we shall prove the following result.

Lemma 6.1. For all $\lambda \in \Lambda$ the operator $\hat{K}(\lambda)$ is compact.

We can now apply the following result, proved by STEINBERG (1968).

LEMMA 6.2. Let $E$ be a Banach space and $\Delta$ a subset of the complex plane which is open and connected. If $T(\lambda)$ is an 
analytic family of compact operators on $E$ for $\lambda \in \Delta$ then either $(I-T(\lambda))$ is nowhere invertible in $\Delta$ or $(I-T(\lambda))^{-1}$ is meromorphic in $\Delta$.

(A function $\phi(\lambda)$ defined on a set $V \subseteq \mathbb{C}$ is called meromorphic if it is analytic on $V$ except for an at most countable set of elements of $V$ which are poles of finite order of $\phi$.) It is clear that $\|\hat{K}(\lambda)\| \rightarrow 0$ if $\operatorname{Re} \lambda \rightarrow \infty$, implying that $I-\hat{K}(\lambda)$ is invertible if $\operatorname{Re} \lambda$ is large enough. Thus lemma 6.1 and lemma 6.2 yield:

THEOREM 6.3. The function $\lambda \rightarrow(I-\hat{K}(\lambda))^{-1}$ is meromorphic in $\Lambda$.

Therefore the set $\Sigma$ defined by (4.3) is a discrete set whose elements are poles of $(I-\hat{K}(\lambda))^{-1}$ of finite order.

Now we shall employ positivity arguments to determine the so-called dominant singular point, i.e. the element of $\Sigma$ with the largest real part. Before doing so we make an additional assumption on the growthrate $g$.

AsSUMPTION 6.4. There exists a $\delta>0$ such that $2 g(x)-g(2 x) \geqslant \delta$, all $x \in[0, \infty)$.

In chapter II a similar assumption has been made to establish compactness of the semigroup. In section 9 we shall explain why assumption 6.4 is imposed. A consequence of this assumption is that a baby cell can not attain arbitrarily small sizes. We shall make this more explicit. If a cell is born with size $x$, then it can divide not earlier than $a_{0}$ time units later, and its daughers can not be smaller than

$$
\gamma(x) \stackrel{\text { def }}{=} \frac{1}{2} X\left(a_{0}, x\right)=\frac{1}{2} G^{-1}\left(a_{0}+G(x)\right) .
$$

LEMMA 6.5. $\gamma$ has precisely one fixed point $x_{0}$. Let for arbitrary $x_{1} \geqslant 0$ the sequence $\left\{x_{n}\right\}$ be defined recursively as $x_{n+1}=\gamma\left(x_{n}\right), n \geqslant 1$ then: $x_{1}<x_{0}$ implies $x_{n}<x_{0}$ and $x_{1}>x_{0}$ implies $x_{n}>x_{0}$, and $\lim _{n \rightarrow \infty} x_{n}=x_{0}$.

Proof. The equation $\gamma(x)=x$ is equivalent to $G(2 x)-G(x)=a_{0}$. The left hand side is zero when $x=0$ and its derivative $\frac{2 g(x)-g(2 x)}{g(2 x) g(x)}>\frac{\delta}{g_{\min }}>0$ by assumptions $\left(A_{g}\right)$ and 6.4. This proves the first part of the lemma.

Next we consider the recurrence relation $x_{n+1}=\gamma\left(x_{n}\right)$. Since $\gamma(0)>0, \gamma$ is continuous, and $x_{0}$ is the unique solution of $x_{0}=\gamma\left(x_{0}\right)$, we have $\gamma(x)>x$ if $0 \leqslant x<x_{0}$ and $\gamma(x)<x$ if $x>x_{0}$. In combination with $\gamma^{\prime}(x)=\frac{g\left(a_{0}+G(x)\right)}{2 g(x)}>0$ this implies $x_{n}<x_{n+1}<x_{n+1}<x_{0}$ if $x_{1}<x_{0}$ and $x_{n}>x_{n+1}>x_{0}$ if $x_{1}>x_{0}$. Therefore $\lim _{n \rightarrow \infty} x_{n}$ exists. The continuity of $\gamma$ implies that it is a fixed point.

From this lemma and the observation that a baby cell attains the minimum birth size if all its ancestors have divided at age $a_{0}$, it follows that this minimum birth size is $x_{0}$ (which is positive if $a_{0}$ is positive), provided that there are infinitely many ancestors who all lived under the same growth regime.

REMARK 6.6. The state space $\Omega_{s}$ referred to in section 1 is given by $\Omega_{s}=\left\{(a, x) \in \mathbb{R}^{+} \times \mathbb{R}^{+} \mid x \geqslant X\left(a, x_{0}\right)\right\}$.

However, we do not want to restrict ourselves à priori to initial data defined on $\Omega_{s}$ only, but admit that $n_{0}(a, x)$ defined in (1.6) is positive on $\Omega \backslash \Omega_{s}$. We can prove the following result.

LEMMA 6.7. If $\psi$ is an eigenvector of $\hat{K}(\lambda)$, then $\psi(x)=0, x<x_{0}$.

PRoof. Let $\psi \in X$. It follows from (6.1) that $\left(\hat{K}(\lambda)^{n} \psi\right)(x)=0$ if $x \leqslant x_{n}$, where $x_{1}=\gamma(0)$ and $x_{n+1}=\gamma\left(x_{n}\right), n \geqslant 1$. If $\psi$ is an eigenvector of $\hat{K}(\lambda)$ then $\psi$ is an eigenvector of $\hat{K}(\lambda)^{n}$ for every positive integer $n$. As a consequence $\psi(x)=0$ if $x \leqslant x_{n}$, and now the result follows from lemma 6.5 .

We denote with $Y$ the subspace of $X$ containing all $\psi \in L_{1}[0, \infty)$ which are identically zero on [0, $\left.x_{0}\right)$. Obviously $\hat{K}(\lambda) Y \subseteq Y$. We let $\hat{K}_{0}(\lambda)$ be the restriction of $\hat{K}(\lambda)$ to $Y$. It is clear immediately that lemma 6.1 and theorem 6.3 remain valid if $\hat{K}(\lambda)$ is replaced by $\hat{K}_{0}(\lambda)$. Moreover (4.3) can be replaced by $\Sigma=\left\{\lambda \in \Lambda \mid 1 \in \sigma\left(\hat{K}_{0}(\lambda)\right)\right\}$. Let $Y_{+}$be the subset of $Y$ containing all elements which are non-negative a.e. (almost everywhere). The following result is straightforward.

THEOREM 6.8. $Y_{+}$defines a cone in $Y$ which is total. Moreover $\hat{K}_{0}(\lambda)$ is positive with respect to $Y_{+}$for all $\lambda \in \Lambda \cap \mathbb{R}$. 
We let $Y_{+}^{*}$ be the dual of $Y_{+}$and this defines a cone in $Y^{\star}$ because $Y_{+}$is total. Clearly $Y_{+}^{*}$ can be identified with $L_{\infty}^{+}\left[x_{0}, \infty\right)$, i.e. all measurable function on $\left[x_{0}, \infty\right)$ which are non-negative and essentially bounded.

The following lemma provides a useful characterization of the non-zero elements of $Y_{+}^{*}$.

LEMMA 6.9. If $F \in Y_{+}^{*}, F \neq 0$, then there exists an $\epsilon>0$ such that for all $f \in Y_{+}$satisfying $f(x)>0$ for almost every $x \in\left[x_{0}+\epsilon, \infty\right)$ the relation $\langle F, f>>0$ holds.

Proof. $F \in Y_{+}^{*}, F \neq 0$ implies that there exists a measurable set $V \subset\left[x_{0}, \infty\right)$ with measure $\mu>0$ such that $F(x)>0, x \in V$. If we choose $\epsilon<\mu$, then the intersection $V \cap\left[x_{0}+\epsilon, \infty\right)$ has a measure which is greater than $\mu-\epsilon>0$, and this yields the result.

Now we can prove the following strong positivity result with respect to $\hat{K}_{0}(\lambda)$.

THEOREM 6.10. For all $\lambda \in \Lambda \cap \mathbb{R}$ the operator $\hat{K}_{0}(\lambda)$ is non-supporting with respect to $Y_{+}$.

PRoof. Let $\psi \in Y_{+}, \psi \neq 0$ and $\lambda \in \Lambda \cap \mathbb{R}$. If we substitute $z=X(-a, 2 x)$ in (6.1) we obtain

$$
\left(\hat{K}_{0}(\lambda) \psi\right)(x)=\int_{x_{0}}^{X\left(-a_{0}, 2 x\right)} e^{-\lambda(G(2 x)-G(z))} \cdot k(G(2 x)-G(z), 2 x) \frac{\psi(z)}{g(z)} d z .
$$

Let $F \in Y_{+}^{*}, F \neq 0$ and let $\epsilon>0$ be given by lemma 6.9. There exists an $x_{1}>x_{0}$ such that $\int_{x_{0}}^{X\left(-a_{0}, 2 x_{1}\right)} \psi(z) d z>0$. This yields that $\left(\hat{K}_{0}(\lambda) \psi\right)(x)>0$ if $x \geqslant x_{1}$. Let $x_{2}=\gamma\left(x_{1}\right)$, where $\gamma$ is defined by (6.2). Then $\left(\hat{K}_{0}(\lambda)^{2} \psi(x)>0\right.$, $x \geqslant x_{2}$. Recursively we find $\left(\hat{K}_{0}(\lambda)^{n} \psi\right)(x)>0, x \geqslant x_{n}$, where $x_{n}=\gamma\left(x_{n-1}\right), n \geqslant 2$. We conclude from lemma 6.5 that there exists a $p \in \mathbb{N}$ such that $x_{n}<x_{0}+\epsilon$ if $n \geqslant p$. Now we can apply lemma 6.9 which says that $<F, \hat{K}_{0}(\lambda)^{n} \psi>0$ if $n \geqslant p$, and this proves the result.

We can draw the following conclusions from theorem 5.2. Let $r_{\lambda}=r\left(\hat{K}_{0}(\lambda)\right), \lambda \in \Lambda$. If $\lambda \in \Lambda \cap \mathbb{R}$, then

(a) $\quad r_{\lambda}$ is an algebraïcally simple eigenvalue of $\hat{K}_{0}(\lambda)$.

(b) The corresponding eigenvector $\psi_{\lambda} \in Y_{+}$satisfies $\psi_{\lambda}(x)>0, x \in\left[x_{0}, \infty\right)$ a.e. We fix $\psi_{\lambda}$ by the normalization $\left\|\psi_{\lambda}\right\|=1$.

(c) The corresponding eigenfunctional $F_{\lambda} \in Y_{+}^{*}$ satisfies $F_{\lambda}(x)>0, x \in\left[x_{0}, \infty\right)$ a.e., i.e. $F_{\lambda}$ is strictly positive. Hence, if $\lambda \in \Lambda$ is real and $r_{\lambda}=1$, then $\lambda \in \Sigma$.

LEMMa 6.11. There exists a unique $\lambda \in \Lambda \cap \mathbb{R}$ such that $r\left(\hat{K}_{0}(\lambda)\right)=1$.

Proof. Let $\lambda, \mu \in \Lambda \cap \mathbb{R}, \lambda>\mu$ and $\psi \in Y_{+}$.

$$
\begin{aligned}
& \left(\hat{K}_{0}(\mu) \psi(x)=\int_{a_{0}}^{G(2 x)} e^{-\mu a} k(a, 2 x) \psi(X(-a, 2 x)) d a\right. \\
& \geqslant e^{(\lambda-\mu) a_{0}} \int_{a_{0}}^{G(2 x)} e^{-\lambda a} k(a, 2 x) \psi(X(-a, 2 x)) d a=e^{(\lambda-\mu) a_{0}}\left(\hat{K}_{0}(\lambda) \psi\right)(x) .
\end{aligned}
$$

If we substitute $\psi=\psi_{\lambda}$, then we obtain $\hat{K}_{0}(\mu) \psi_{\lambda} \geqslant e^{(\lambda-\mu) a_{0}} r_{\lambda} \psi_{\lambda}$. Taking duality pairings with $F_{\mu}$ on both sides yields

$$
r_{\mu} \geqslant e^{(\lambda-\mu) a_{0}} \cdot r_{\lambda}
$$

where we have used that $\left\langle F_{\mu}, \psi_{\lambda} \gg 0\right.$. Thus $\lambda \rightarrow r\left(\hat{K}_{0}(\lambda)\right)$ is strictly decreasing in $\Lambda \cap \mathbb{R}$. Moreover this function is continuous. It follows easily that $\lim _{\lambda \rightarrow \infty} r\left(\hat{K}_{0}(\lambda)\right)=0$. If we can prove that $\lim _{\downarrow \downarrow-d_{\infty}} r\left(\hat{K}_{0}(\lambda)\right)=\infty$ then the conclusion of the lemma follows. We have to distinguish between two cases.

(a) $\quad d_{\infty}=\infty$. Then (6.3) implies that $\lim _{\lambda \rightarrow-\infty} r\left(\hat{K}_{0}(\lambda)\right)=\infty$.

(b) $\quad d_{\infty}<\infty$. Since $\left\|\psi_{\lambda}\right\|=1$,

$$
\begin{aligned}
& r\left(\hat{K}_{0}(\lambda)\right)=\left\|\hat{K}_{0}(\lambda) \psi_{\lambda}\right\|=\int_{x_{0}}^{\infty}\left\{\int_{0}^{\infty} e^{-\lambda t}\left(K(t) \psi_{\lambda}\right)(x) d t\right\} d x=\int_{0}^{\infty} e^{-\lambda t}\left\{\int_{x_{0}}^{\infty}\left(K(t) \psi_{\lambda}\right)(x) d x\right\} d t \\
& =\int_{0}^{\infty} e^{-\lambda t}\left\|K(t) \psi_{\lambda}\right\| d t \geqslant \int_{T_{0}}^{\infty} e^{-\lambda t}\left\|K(t) \psi_{\lambda}\right\| d t \geqslant \int_{T_{0}}^{\infty} m_{K} e^{-d_{\infty} t} e^{-\lambda t} d t=\frac{m_{K}}{\lambda+d_{\infty}} e^{-\left(\lambda+d_{\infty}\right) T_{0}},
\end{aligned}
$$


where we have used lemma 3.1. The change of order of integration was permitted because of Fubini's theorem (DuNFORD \& SCHWARTZ (1958)). It follows that $\lim _{\lambda \downarrow-d_{\infty}} r\left(\hat{K}_{0}(\lambda)\right)=\infty$.

We denote the unique solution of $r\left(\hat{K}_{0}(\lambda)\right)=1$ by $\lambda_{d}$, and we shall write $\psi_{d}$ and $F_{d}$ in stead of $\psi_{\lambda_{d}}$ and $F_{\lambda_{d}}$ respectively. We assume that $\psi_{d}$ and $F_{d}$ are normalized by

$$
\left\|\psi_{d}\right\|=1,\left\langle F_{d}, \psi_{d}\right\rangle=1
$$

In order to prove that indeed $\lambda_{d}$ is indeed the element of $\Sigma$ with the largest real part, we need the following lemma (e.g. Rudin (1966)).

LEMMa 6.12. Let $f \in L_{1}[0, \infty)$ be a complex-valued function. Then $\left|\int_{0}^{\infty} f(x) d x\right|=\int_{0}^{\infty}|f(x)| d x$ if and only if there exists a constant $\alpha \in \mathbb{C},|\alpha|=1$ such that $|f(x)|=\alpha f(x)$ a.e. on $[0, \infty)$.

Theorem 6.13. If $\lambda \in \Sigma, \lambda \neq \lambda_{d}$, then $\operatorname{Re} \lambda<\lambda_{d}$.

Proof. Suppose $\lambda \in \Sigma$ and $\hat{K}_{0}(\lambda) \psi=\psi$. Hence $\left|\hat{K}_{0}(\lambda) \psi\right|=|\psi|$, where $|\psi|(x) \stackrel{\text { def }}{=}|\psi(x)|$. This yields $\hat{K}_{0}\left(\lambda_{R}\right)|\psi| \geqslant|\psi|$, where $\lambda_{R}=\operatorname{Re} \lambda$. Taking duality pairings with $F_{\lambda_{R}}$ on both sides yields $r_{\lambda_{R}}\left\langle F_{\lambda_{R}},|\psi|\right\rangle \geqslant\left\langle F_{\lambda_{R}},|\psi|\right\rangle$, from which we conclude that $r_{\lambda_{R}} \geqslant 1$. In the proof of lemma 6.11 we have shown that $\lambda \rightarrow r_{\lambda}$ is decreasing in $\lambda \in \Lambda \cap \mathbb{R}$, and this implies that $\lambda_{R}=\operatorname{Re} \lambda_{d}$. Now suppose that $\operatorname{Re} \lambda=\lambda_{d}$ and $\operatorname{Im} \lambda=\eta$. Thus $\hat{K}_{0}\left(\lambda_{d}\right)|\psi| \geqslant|\psi|$. Suppose that $\hat{K}_{0}\left(\lambda_{d}\right)|\psi|>|\psi|$. Taking duality pairings with $F_{d}$ on both sides yields $\left.\left\langle F_{d},|\psi|\right\rangle\right\rangle\left\langle F_{d},|\psi|\right\rangle$ which is a contradiction. As a consequence $\hat{K}_{0}\left(\lambda_{d}\right)|\psi|=|\psi|$, from which we deduce that $|\psi|=c \cdot \psi_{d}$ for some constant $c$ which we may assume to be one. Therefore $\psi(x)=\psi_{d}(x) e^{j \alpha(x)}$ for some real-valued function $\alpha$. If we substitute this in $\hat{K}_{0}\left(\lambda_{d}\right) \psi_{d}=\left|\hat{K}_{0}(\lambda) \psi\right|$ we obtain

$$
\int_{a_{0}}^{\infty} e^{-\lambda_{d} a} k(a, 2 x) \psi_{d}(X(-a, 2 x)) d a=\left|\int_{a_{0}}^{\infty} e^{-\lambda_{d} a-i \eta a} k(a, 2 x) \psi_{d}(X(-a, 2 x)) e^{i \alpha(X(-a, 2 x))} d a\right| .
$$

From lemma 6.12 we conclude that $\alpha(X(-a, 2 x))-\eta a=\beta$, for some constant $\beta$. If we substitute this in $\hat{K}_{0}(\lambda) \psi=\psi$ we obtain $\left.e^{i \beta} \int_{0}^{\infty} e^{-\lambda_{d} a} k(a, 2 x)\right) d a=\psi_{d}(x) e^{i \alpha(x)}$, thus $\alpha(x)=\beta$ from which we conclude that $\eta=\operatorname{Im} \lambda=0$.

This result, combined with the Riemann-Lebesgue lemma (lemma 4.3) and theorem 6.3, implies among others that there exists a positive horizontal distance between $\lambda_{d}$ and the other points in $\Sigma$.

COROLLARY 6.14. There exists an $\epsilon>0$ such that $\lambda_{d}-\epsilon>-d_{\infty}$ and $\operatorname{Re} \lambda \leqslant \lambda_{d}-\epsilon$ if $\lambda \in \Sigma, \lambda \neq \lambda_{d}$.

Clearly $\hat{K}_{0}(\lambda)$ and $\hat{K}(\lambda)$ have the same eigenvectors (lemma 6.7). However $\hat{K}_{0}(\lambda)^{*}$ and $\hat{K}(\lambda)^{*}$ do not have the same eigenvectors. Let $F_{d}^{\prime}$ be the eigenvector of $\hat{K}\left(\lambda_{d}\right)^{*}$ corresponding to the eigenvalue one. Obviously, $F_{d}^{\prime}$ defines a positive functional on $X$. We can prove the following relation between $F_{d}$ and $F_{d}^{\prime}$. Let $\left\langle F_{d}^{\prime}, \psi_{d}\right\rangle=1$.

THEOREM 6.15. For all $\psi \in Y$, the equality $\left\langle F_{d}, \psi\right\rangle=\left\langle F_{d}^{\prime}, \psi\right\rangle$ holds.

Proof. Let $\psi \in Y$, then $\psi=\left\langle F_{d}, \psi\right\rangle \cdot \psi_{d}+\rho$, where $\rho \in \mathcal{R}\left(\hat{K}_{0}\left(\lambda_{d}\right)-I\right) \stackrel{\text { def }}{=} Z$, i.e. the range of $\hat{K}_{0}\left(\lambda_{d}\right)-I$. Since the spectral radius of the restriction of $\hat{K}_{0}\left(\lambda_{d}\right)$ to the subspace $Z$ is strictly less than one (theorem $5.2 \mathrm{~d}$ ) it follows that $\left\|\hat{K}_{0}\left(\lambda_{d}\right)^{n} \rho\right\|<\theta^{n}\|\rho\|$ for all $\rho \in Z$, where $\theta$ is some constant strictly less than one. Since $\hat{K}\left(\lambda_{d}\right) \psi=K_{0}\left(\lambda_{d}\right) \psi$ we have $\left\langle F_{d, \psi}^{\prime}\right\rangle=\left\langle\hat{K}\left(\lambda_{d}\right)^{*_{n}} F_{d}^{\prime}, \psi\right\rangle=\left\langle F_{d}^{\prime}, \hat{K}_{0}\left(\lambda_{d}\right)^{n}\left(\left\langle F_{d}, \psi\right\rangle \psi_{d}+\rho\right)\right\rangle=\left\langle F_{d}, \psi\right\rangle+\left\langle F_{d}^{\prime}, \hat{K}_{0}\left(\lambda_{d}\right)^{n} \rho\right\rangle$. If we let $n \rightarrow \infty$ then the second term at the right-hand-side tends to zero yielding that $\left\langle F_{d}^{\prime}, \psi\right\rangle=\left\langle F_{d}, \psi\right\rangle$.

\section{Computation of the residue in $\lambda_{d}$.}

Here we shall concentrate on the behaviour of $(I-\hat{K}(\lambda))^{-1}$ in a neighbourhood of $\lambda=\lambda_{d}$, which is a pole of finite order (cf. theorem 6.3). The techniques exploited in this section are very similar to those in a paper by SCHUMrTZKY \& WeNSKa (1975). We define

$$
R(\lambda)=(I-\hat{K}(\lambda))^{-1}, \quad \lambda \in \Lambda \backslash \Sigma .
$$

Since $\hat{K}(\lambda)$ is analytic in a neighbourhood of $\lambda_{d}$ we can write down its Taylor expansion.

$$
\hat{K}(\lambda)=\sum_{n=0}^{\infty}\left(\lambda-\lambda_{d}\right)^{n} K_{n},
$$


where the series converges in the norm topology. Let $p \geqslant 1$ be the order of the pole of $R(\lambda)$ in $\lambda=\lambda_{d}$. In a neighbourhood of $\lambda_{d}, R(\lambda)$ can be represented by a Laurent series:

$$
R(\lambda)=\sum_{n=-p}^{\infty}\left(\lambda-\lambda_{d}\right)^{n} R_{n}
$$

where by definition $R_{-p} \neq 0$. From

$$
R(\lambda)(I-\hat{K}(\lambda))=(I-\hat{K}(\lambda)) R(\lambda)=I
$$

if follows immediately that

$$
R_{-p}\left(I-K_{0}\right)=\left(I-K_{0}\right) R_{-p}=0 .
$$

From this relation and $K_{0}=\hat{K}\left(\lambda_{d}\right)$ we obtain

$$
\mathscr{R}\left(R_{-p}\right)=\left\{\psi_{d}\right\}
$$

where $\mathscr{R}\left(R_{-p}\right)$ denotes the range of the operator $R_{-p}$, and $\left\{\psi_{d}\right\}$ stands for the span of the positive eigenvector $\psi_{d}$, i.e. $\left\{\psi_{d}\right\}=\left\{\gamma \cdot \psi_{d} \mid \gamma \in \mathbb{C}\right\}$. A relation similar to (7.4) is valid for the dual operators $K_{0}^{*}=\hat{K}\left(\lambda_{d}\right)^{*}$ and $R_{-p}^{*}$. Therefore

$$
\mathscr{R}\left(R_{-p}^{*}\right)=\left\{F_{d}\right\}
$$

From (7.4) we also deduce that

$$
\begin{aligned}
& -R_{-p} K_{1}+R_{-p+1}\left(I-K_{0}\right)=0, \text { if } p>1, \\
& -R_{-1} K_{1}+R_{0}\left(I-K_{0}\right)=I, \quad \text { if } p=1 .
\end{aligned}
$$

Together with (7.5) this implies

$$
\begin{aligned}
& R_{-p} K_{1} R_{-p}=0, \text { if } p>1, \\
& R_{-1} K_{1} R_{-1}=-R_{-1}, \text { if } p=1 .
\end{aligned}
$$

We can state our main result now.

THEOREM 7.1. $R(\lambda)$ has a pole of order one in $\lambda=\lambda_{d}$ and the residue $R_{-1}$ is given by

$$
R_{-1} \psi=\frac{\left.\left\langle F_{d}^{\prime},\right\rangle\right\rangle}{\left\langle F_{d}^{\prime},-K_{1} \psi_{d}\right\rangle} \cdot \psi_{d}, \psi \in X
$$

Observe that $-K_{1}=\left[-\frac{d}{d \lambda} \hat{K}(\lambda)\right]_{\lambda=\lambda_{d}}$ defines a positive non-supporting operator on $Y$ and thus it follows from theorem 6.15 that $\left\langle F_{d}^{\prime},-K_{1} \psi_{d}\right\rangle=\left\langle F_{d},-K_{1} \psi_{d}\right\rangle>0$.

Proof OF THEOREM 7.1. Let $\phi_{d}$ and $H_{d}$ be solutions of $R_{-p} \phi=\psi_{d}$ and $R_{-p}^{*} H=F_{d}$ respectively. On account of (7.6) and (7.7) such solutions indeed exist. If $p\rangle 1$ then (7.9a) yields $0=\left\langle H_{d}, R_{-p} K_{1} R_{-p} \phi_{d}\right\rangle=\left\langle F_{d}, K_{1} \psi_{d}\right\rangle$ which is a contradiction since $F_{d}$ is strictly positive and $-K_{1} \psi_{d}$ is positive and nonzero. Therefore $p=1$, and $\Re\left(R_{-1}\right)=\left\{\psi_{d}\right\}$. Now let $R_{-1} \psi=f(\psi) \cdot \psi_{d}$ for some linear functional $f$. Then $\left\langle H_{d}, R-1 \psi\right\rangle=$ $\left\langle R_{-1}^{*} H_{d}, \psi\right\rangle=\left\langle F_{d}, \psi\right\rangle=\left\langle H_{d},-R_{-1} K R_{-1} \psi\right\rangle=\left\langle R_{-1}^{*} H_{d},-K_{1}\left(f(\psi) \cdot \psi_{d}\right)\right\rangle=f(\psi) \cdot\left\langle F_{d},-K_{1} \psi_{d}\right\rangle$, thus $f(\psi)=\left\langle F_{d}, \psi\right\rangle /\left\langle F_{d},-K_{1} \psi_{d}\right\rangle$ which proves the result.

It is not à priori clear whether or not $\left\langle F_{d}^{\prime}, \psi\right\rangle>0$ if $\psi \in X_{+}, \psi \neq 0$. This, however, is proved in the following lemma.

LEMMA 7.2. If $\psi \in X_{+}, \psi \neq 0$ then $\left\langle F_{d}^{\prime}, \psi \geqslant 0\right.$.

PRoor. If the restriction of $\psi$ to $\left[x_{0}, \infty\right)$ is not identically zero, then the result follows from theorem 6.15 . Now suppose that $\psi$ is positive on a subset of $\left[0, x_{0}\right]$ with positive measure. Thus

$\left(\hat{K}\left(\lambda_{d}\right) \psi\right)(x) \geqslant \int_{G(2 x)-G\left(x_{0}\right)}^{G(2 x)} e^{-\lambda_{d} a} k(a, 2 x) \psi(X(-a, 2 x)) d a=\int_{0}^{x_{0}} e^{-\lambda_{d}(G(2 x)-G(z))} \cdot k(G(2 x)-G(z), 2 x) \frac{\psi(z)}{g(z)} d z>0$

for all $x \geqslant x_{0}$. Therefore $\left\langle F_{d}^{\prime}, \psi\right\rangle=\left\langle\hat{K}\left(\lambda_{d}\right)^{*} F_{d}^{\prime}, \psi\right\rangle=\left\langle F_{d}^{\prime}, \hat{K}\left(\lambda_{d}\right) \psi\right\rangle>0$. 


\section{The inverse Laplace transform}

Let $E$ be a Banach space. The Hardy-Lebesgue class $H_{p}(\alpha ; E)$ is the class of functions $g(\lambda)$ with values in $E$, which are analytic in $\operatorname{Re} \lambda>\alpha$ and satisfy the following conditions (cf. Friedman \& Shinbrot (1967), Hille \& Phillips (1957)).

$$
\begin{aligned}
& \sup _{\zeta>\alpha}\left\{\int_{-\infty}^{\infty}\|g(\zeta+i \eta)\|^{p} d \eta\right\}^{\frac{1}{p}}<\infty, \\
& g(\alpha+i \eta)=\lim _{\zeta \downarrow \alpha} g(\zeta+i \eta) \text { exists a.e. and is an element of } L_{p}(-\infty, \infty ; E) .
\end{aligned}
$$

The following inverse Laplace transform formula can be found in FRIEDMAN \& SHINBROT (1967).

LEMMA 8.1. Let $g(\lambda) \in H_{1}(\alpha ; E)$, then the function

$$
f(t)=\frac{1}{2 \pi i} \int_{\gamma-i \infty}^{\gamma+i \infty} e^{\lambda t} g(\lambda) d \lambda, \quad(\gamma \geqslant \alpha)
$$

is defined and independent of $\gamma$, for all $t \in(-\infty, \infty)$. Moreover $f(t)=0, t<0, f(t)$ is continuous and $\hat{f}(\lambda)=g(\lambda)$.

We rewrite the abstract renewal equation (2.17) as

$$
B=\Phi+K \star B,
$$

where $K \star B$ denotes the convolution product, i.e. $(K \star B)(t)=\int_{0}^{t} K(a) B(t-a) d a$. If we substitute

$$
B=\Phi+v,
$$

we obtain

$$
v=\Psi+K \star v,
$$

where

$$
\Psi=K \star \Phi .
$$

Taking Laplace transforms on both sides of (8.5) gives us

$$
\hat{v}(\lambda)=(I-\hat{K}(\lambda))^{-1} \hat{\Psi}(\lambda)
$$

We can prove the following result.

LEMMA 8.2. $\hat{v}(\lambda) \in H_{1}(\alpha ; X)$, if $\alpha>\lambda_{d}$.

Proof. Let $\lambda \in \mathbb{C}$ be such that $\operatorname{Re} \lambda \geqslant \alpha$. The function $\eta \rightarrow \hat{\Psi}(\zeta+i \eta)$ is an element of $L_{1}(-\infty, \infty ; X)$ if $\zeta>-d_{\infty}$ (see section 6.3 of Hille \& PHILlips (1957)). Moreover we know from the Riemann-Lebesgue lemma (lemma 4.3) that $\left\|(I-\hat{K}(\zeta+i \eta))^{-1}\right\| \leqslant 2$ if $|\eta|$ is large enough, say $|\eta| \geqslant \eta_{0}$. From the continuity of the function $\eta \rightarrow(I-\hat{K}(\zeta+i \eta))^{-1}$ on $\left[-\eta_{0}, \eta_{0}\right]$ (if $\zeta \geqslant \alpha$ ) we conclude that there exists a constant $C>0$ such that $\left\|(I-\hat{K}(\zeta+i \eta))^{-1}\right\|<C$ for all $\eta \in(-\infty, \infty)$. Thus $\|\hat{v}(\zeta+i \eta)\| \leqslant C\|\hat{\Psi}(\zeta+i \eta)\|$ where we have used (8.7). The positivity of $K(t)$ and $\Psi(t)$ yields that

$$
\|\hat{\Psi}(\zeta+i \eta)\| \leqslant\|\hat{\Psi}(\alpha+i \eta)\|, \quad \zeta \geqslant \alpha
$$

and we conclude that condition (8.1a) is satisfied. The validity of condition (8.1b) follows from the analyticity of $(I-\hat{K}(\lambda))^{-1}, \hat{\Phi}(\lambda)$ and $\hat{K}(\lambda)$ on the region $\operatorname{Re} \lambda>\lambda_{d}$ and the fact that $\alpha>\lambda_{d}$. 


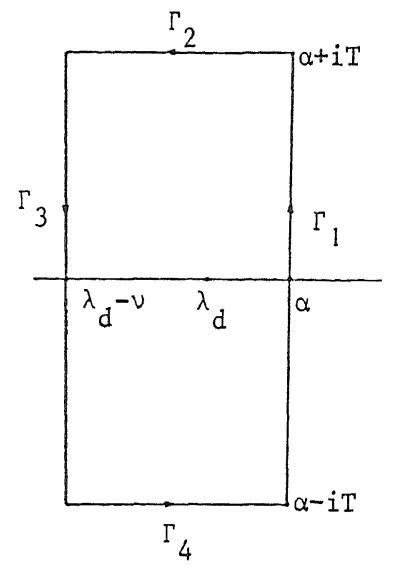

Figure 8.1. $\Gamma=\bigcup_{i=1}^{4} \Gamma_{i}$

Now let $\alpha>\lambda_{d}$, then lemma 8.1 yields that

$$
v(t)=\frac{1}{2 \pi i} \int_{\alpha-i \infty}^{\alpha+i \infty} e^{\lambda t} \hat{v}(\lambda) d \lambda
$$

is well-defined. Some contributions to this integral can be evaluated by the method of residues. Therefore we shift the vertical integration curve $\operatorname{Re} \lambda=\alpha$ to the left across the singularity $\lambda=\lambda_{d}$, such that it crosses no other elements of $\Sigma$ (see fig. 8.1). Let $\epsilon>0$ be given by corollary 6.14 , and let $0<\nu<\epsilon$. Let $\Gamma$ be the rectangular contour in fig. 2 . It follows immediately from the Riemann-Lebesgue lemma (lemma 4.3) that

$$
\lim _{T \rightarrow \infty} \int_{\Gamma_{i}} e^{\lambda t} \hat{v}(\lambda) d \lambda=0, \quad i=2,4
$$

Now it follows from Cauchy's theorem (which is also valid for vector-valued functions: see Hille \& PHILLIPS (1957)) that

$$
\nu(t)=\frac{1}{2 \pi i} \oint_{\Gamma} e^{\lambda t} \hat{v}(\lambda) d \lambda+\frac{1}{2 \pi i} \lim _{T \rightarrow \infty} \int_{\lambda_{d}-\nu-i T}^{\lambda_{d}-\nu+i T} e^{\lambda t} \hat{v}(\lambda) d \lambda,
$$

where we have used that the first integral does not depend on $T$. The residue theorem gives:

$$
\begin{aligned}
& \frac{1}{2 \pi i} \oint_{\Gamma} e^{\lambda t} \hat{v}(\lambda) d \lambda=\operatorname{Res}_{\lambda=\lambda_{d}}\left\{e^{\lambda t} \hat{v}(\lambda)\right\}=e^{\lambda_{d} t} R_{-1} \hat{\Psi}\left(\lambda_{d}\right)=e^{\lambda_{d} t} R_{-1} \hat{K}\left(\lambda_{d}\right) \hat{\Phi}\left(\lambda_{d}\right)=e^{\lambda_{d} t} \cdot \frac{\left\langle F_{d}^{\prime}, \hat{K}\left(\lambda_{d}\right) \hat{\Phi}\left(\lambda_{d}\right)\right\rangle}{\left\langle F_{d}^{\prime},-K_{1} \psi_{d}\right\rangle} \cdot \psi_{d} \\
& =e^{\lambda_{d} t} \frac{\left\langle F_{d}^{\prime}-K_{1} \hat{\Phi}\left(\lambda_{d}\right)\right\rangle}{\left\langle F_{d}^{\prime},-K_{1} \psi_{d}\right\rangle} \cdot \psi_{d},
\end{aligned}
$$

where we have used theorem 7.1, (8.6) and (8.7). As in the proof of lemma 8.2 we have that the function $\eta \rightarrow \hat{\nu}\left(\lambda_{d}-v+i \eta\right)$ is an element of $L_{1}(-\infty, \infty ; X)$. Now

$$
\left\|\frac{1}{2 \pi i} \int_{\lambda_{d}-\nu-i \infty}^{\lambda_{d}-\nu+i \infty} e^{\lambda t} \hat{v}(\lambda) d \lambda\right\| \leqslant M \cdot e^{\left(\lambda_{d}-\nu\right) t},
$$

where

$$
M \stackrel{d e f}{=} \frac{1}{2 \pi} \int_{-\infty}^{\infty}\left\|\hat{v}\left(\lambda_{d}-\nu+i \eta\right)\right\| d \eta \text { depends on } \nu \text { and } \Phi .
$$

We can state our main result now.

COROLlaRY 8.3. Let $\epsilon>0$ be given by corollary 6.14, and let $0<\nu<\epsilon$, then $\left\|e^{-\lambda_{d} t} B(t)-c \cdot \psi_{d}\right\| \leqslant L e^{-\nu t}, t \geqslant 0$, for 
some constant $L$, where $c=\frac{\left\langle F_{d}^{\prime}, \hat{\Phi}\left(\lambda_{d}\right)\right\rangle}{\left\langle F_{d}^{\prime},-K_{1} \psi_{d}\right\rangle}$ is a constant depending linearly on $\Phi$.

PRoof. We have $B(t)=\Phi(t)+v(t)$, and $v(t)=e^{\lambda_{d} t}\left(c \cdot \psi_{d}+O\left(e^{-\nu t}\right)\right)$. Now the result follows from lemma 3.1.

REMARK 8.4. Observe from corollary 8.3 that if $t$ has become infinite, no cells with size less than $x_{0}$ are born, although such cells may be present at time zero.

\section{Interpretation, conclusions and final remarks}

For the sake of converience we repeat (2.11) and (2.12)

$$
\begin{aligned}
& n(t, a, x)=\frac{g(X(-t, x))}{g(x)} Q(t, a-t, X(-t, x)) n_{0}(a-t, X(-t, x)), t \leqslant a, \\
& n(t, a, x)=\frac{g(X(-a, x))}{g(x)} E(a, X(-a, x)) B(t-a, X(-a, x)), t>a .
\end{aligned}
$$

This does not define a classical solution of (1.4)-(1.6). However, it can be proved that $n$ is differentiable along the characteristics of the partial differential operator $D=\frac{\partial}{\partial t}+\frac{\partial}{\partial a}+g(x) \frac{\partial}{\partial x}$, and in this sense indeed is a solution of (1.4)-(1.6).

Let

$$
n_{d}(a, x)=e^{-\lambda_{d} a} \cdot \frac{g(X(-a, x))}{g(x)} E(a, X(-a, x)) \psi_{d}(X(-a, x)) .
$$

Now we can restrate corollary 8.3 in terms of the solution $n$ of (1.4)-(1.6).

COROLlary 9.1. Let $\epsilon>0$ be given by corollary 6.14 and let $0<\nu<\epsilon$, then the solution $n(t, a, x)$ of (1.4)-(1.6) satisfies $\left\|e^{-\lambda_{d^{t}}} n(t, \cdot, \cdot)-h\left(n_{0}\right) \cdot n_{d}\right\| \leqslant L^{\prime} e^{-\nu t}\left\|n_{0}\right\|, t \geqslant 0$, where $\|\cdot\|$ stands for the $L_{1}(\Omega)-n o r m, L^{\prime}$ is a positive constant, and $h$ is a strictly positive linear functional on $L_{1}(\Omega)$.

REMARK 9.2. $h$ can be computed from $h\left(n_{0}\right)=\frac{\left\langle F_{d}^{\prime}, \hat{\Phi}\left(\lambda_{d}\right)\right\rangle}{\left\langle F_{d}^{\prime},-K_{1} \psi_{d}\right\rangle}$.

Corollary 9.1 is a typical renewal result. The population grows (or decays) exponentially with exponent $\lambda_{d}$ (which is sometimes called the Malthusian parameter). As time increases an asymptotically stable age-size distribution is reached. If $t=\infty$ the dependence on the initial condition is only reflected by the scalar $h\left(n_{0}\right)$.

If in our model the rates $b$ and $\mu$ depend on age only then we can integrate (1.4)-(1.6) over all sizes $x$ and we find the age-dependent problem

$$
\begin{aligned}
& \frac{\partial N}{\partial t}+\frac{\partial N}{\partial a}=-(\mu(a)+b(a)) N(t, a) \\
& N(t, 0)=2 \int_{0}^{\infty} b(a) N(t, a) d a \\
& N(0, a)=N_{0}(a)
\end{aligned}
$$

where $N(t, a) \stackrel{\text { def }}{=} \int_{0}^{\infty} n(t, a, x) d x$. If the assumptions $\left(A_{b}\right),\left(A_{\mu}\right)$ and $\left(A_{d}\right)$ of section 1 are satisfied then a stable agedistribution is reached as $t \rightarrow \infty$ :

$$
N(t, a) \sim e^{\lambda_{d} t} N_{d}(a), t \rightarrow \infty,
$$

(this result can also be found in EISEN (1979)) and the growthrate $g(x)$ has no effect on this stable age-distribution. More details can be found in HANNSGEN et al. (1985).

Now we shall explain what can happen if assumption 6.4 is not fulfilled.

I. We expect that most of our result remain valid if $g(2 x)<2 g(x)$, all $x$ (but not necessarily $2 g(x)-g(2 x)>\delta$, for some $\delta>0$ ). But probably one gets involved with great technical difficulties, which, however, do not provide additional insight. 
II. If $g(2 x)>2 g(x)$, for all $x$, then some sort of instability comes into the problem. Although $\gamma$ defined by (6.2) again has a unique fixed point $x_{0}$, in this case it is unstable:

$$
\left.\frac{d \gamma}{d x}\right|_{x=x_{0}}=\frac{g\left(2 x_{0}\right)}{2 g\left(x_{0}\right)}>1
$$

For the sequence $\left\{x_{n}\right\}$ of lemma 6.4 this result in

$$
\begin{aligned}
& x_{n} \rightarrow 0, \text { if } x_{1}<x_{0}, \\
& x_{n} \rightarrow \infty, \text { if } x_{1}>x_{0} .
\end{aligned}
$$

If we start with a population all of whose members have size $>\bar{x}(0)$, where $\bar{x}(0)>x_{0}$, then at time $t$ all individuals have size $>\bar{x}(t)$, where $\bar{x}(t) \rightarrow \infty$. As a consequence there cannot exist a stable age-size distribution. A second problem arising in this case is caused by the fact that growth becomes very small if $x$ tends to zero. As a consequence individuals can not grow away from zero.

III. Suppose that $g(2 x)=2 g(x)$, all $x$. (Notice that this and also the former case is actually excluded by the boundedness condition on $g$. However, the same integral equation for the birth function $B(t)$ still holds.) Biologically this condition means that the time $T$ needed to grow from $x$ to $2 x$ does not depend on $x$. We can prove that in this case the set of singular points $\Sigma$ is periodic, i.e. there exists a $p>0$ such that $\lambda \in \Sigma \Rightarrow \lambda+i k p \in \Sigma, k \in \mathbb{Z}$ (compare II.5).

LEMMA 9.3. Let $g(2 x)=2 g(x)$, for all $x$ and let $T=G(2 x)-G(x)$ (which does not depend on $x$ ), then $\sum$ is periodic with period $p=\frac{2 \pi}{T}$.

Proof. Suppose $\lambda \in \Sigma$ and let $\psi \in X$ be determined by $\hat{K}(\lambda) \psi=\psi$ :

$$
\begin{aligned}
& \qquad(x)=\int_{a_{0}}^{\infty} e^{-\lambda a} k(a, 2 x) \psi(X(-a, 2 x)) d a \\
& \text { Let } T=G(2 x)-G(x) \text { and } p=\frac{2 \pi}{T} \text {. Let } \psi_{k}(x)=e^{-i k p G(x)} \cdot \psi(x) \text {, then } \\
& \left.\qquad \hat{K}(\lambda+i k p) \psi_{k}\right)(x)=\int_{a_{0}}^{\infty} e^{-\lambda a} e^{-i k p a} k(a, 2 x) \psi(X(-a, 2 x)) e^{-i k p(G(2 x)-a)} d a \\
& =e^{-i k p G(2 x)} \int_{a_{0}}^{\infty} e^{-\lambda a} k(a, 2 x) \psi(X(-a, 2 x)) d a=e^{-i k p(T+G(x))} \cdot \psi(x)=\psi_{k}(x),
\end{aligned}
$$

hence $\lambda+i k p \in \Sigma$.

Now let $\psi_{k}(x)=e^{-i k p G(x)} \cdot \psi_{d}(x)$, where $\psi_{d}$ is the positive eigenvector of $\hat{K}\left(\lambda_{d}\right)$ (assumed that a solution $\lambda_{d}$ of $r(\hat{K}(\lambda))=1$ exists). Let

$$
n_{0}^{k}(a, x)=e^{-\lambda_{k} a} \frac{g(X(-a, x))}{g(x)} E(a, X(-a, x)) \cdot \psi_{k}(X(-a, x)), \quad k \in \mathbb{Z},
$$

where $\lambda_{k}=\lambda_{d}+i k p$ (see (9.1)). Choose $\gamma_{k} \in \mathbb{C}, k \in \mathbb{Z}$ such that $\Sigma_{k=1}^{\infty}\left|\gamma_{k}\right|<\frac{1}{2}, \gamma_{-k}=\bar{\gamma}_{k}$, and define the initial age-size-distribution $n_{0}(a, x)$ by

$$
n_{0}(a, x) \stackrel{\text { def }}{=} n_{0}^{0}(a, x)+\sum_{k=1}^{\infty} \gamma_{k} n_{0}^{k}(a, x)=\left(1+2 R e \sum_{k=1}^{\infty} \gamma_{k} e^{-i k p G(x)}\right) \cdot n_{0}^{0}(a, x),
$$

then $n_{0}(a, x) \geqslant 0,(a, x) \in \Omega$ and the solution $B(t, x)$ of the associated integral equation (2.14) is given by

$$
B(t, x)=e^{\lambda_{d} t} \cdot \psi_{d}(x) \cdot\left\{1+2 \operatorname{Re} \sum_{k=1}^{\infty} \gamma_{k} e^{i k p(t-G(x))}\right\}=e^{\lambda_{d} t} \cdot \psi_{d}(x) \cdot h(t, x)
$$

where

$$
h(t, x) \stackrel{\text { def }}{=} 1+2 \operatorname{Re} \sum_{k=1}^{\infty} \gamma_{k} e^{i k p(t-G(x))}
$$

satisfies 


$$
\begin{aligned}
& h(t+T, x)=h(t, x), \\
& h(t, 2 x)=h(t, x) .
\end{aligned}
$$

This proves that there is no convergence to a stable age-size-distribution in this case (compare II.11).

This result disproves a remark of BeLL (1968) which says that in case of exponential growth $(g(x)=c \cdot x)$ there can exist a stable age-size-distribution if $b$ depends in an appropriate manner on $x$ and $a$. TRUCCO \& BeLL (1970) showed that in the case of dispersionless growth (i.e. $\frac{1}{x} X(a, x)$ depends on a only: this is satisfied if $g(x)=c \cdot x)$ it is not possible that the first and second moments of the distribution of birth sizes both approach finite non-zero limits as $t \rightarrow \infty$, yielding that there does not exist a stable age-size distribution (see also Trucco (1970)). Hannsgen, Tyson \& Watson (1985) proved that in case of exponential growth and under the assumption that the generation time (= age at which a cell divides) is a random variable with a given probability density function there cannot exist a stable, time-independent size distribution for the birth function.

IV. If $[0, \infty)=I_{1} \cup I_{2} \cup I_{3}$ such that $g(2 x)<2 g(x), x \in I_{1}, g(2 x)=2 g(x), \quad x \in I_{2}, g(2 x)>2 g(x)$, $x \in I_{3}$, then the question of convergence to a stable distribution is a very hard one, but also a very interesting and exciting one from the mathematical point of view.

The reason for making assumption $\left(A_{d}\right)$ is a technical one. It guarantees the existence of a dominant element $\lambda_{d}$ of $\Sigma$ (see lemma 6.11).

Undoubtedly our theory is also valid if a less restrictive condition than $\left(A_{g}\right)$ is imposed. However, our main purpose is not generality but to give an idea how abstract results from functional analysis can be used in the study of concrete structured population models. The results that we obtained here can also be found using semigroup methods, and readers who are trying to do so, will find out that the two approaches are more closely linked then it seems at first sight.

\section{Appendix}

Here we shall prove that for all $\lambda \in \Lambda$ the operator $\hat{K}(\lambda)$ is compact. We need the following result of KRASNOSELSKII et al. (1976, chapter $2, \S 5.6)$. They proved that a linear integral operator which has a compact majorant is compact itself. We shall make this more precise. Let $\Omega \subseteq \mathbb{R}$ be a measurable set and let the linear integral operator $T: L_{1}(\Omega) \rightarrow L_{1}(\Omega)$ be given by

$$
(T \phi)(x)=\int_{\Omega} h(x, y) \phi(y) d y .
$$

Suppose that

$$
|h(x, y)| \leqslant h^{+}(x, y), x, y \in \Omega,
$$

and let the operator $T^{+}$be given by

$$
\left(T^{+} \phi\right)(x)=\int_{\Omega} h^{+}(x, y) \phi(y) d y .
$$

Then the following result holds (KRASNOSELSKII et al. (1976)):

LEMMA 1. If $T^{+}$is a bounded, compact operator from $L_{1}(\Omega)$ into itself then $T$ is also compact.

Now let $\lambda \in \Omega$, then

$$
\hat{K}(\lambda) \psi(x)=\int_{0}^{X\left(-a_{0}, 2 x\right)} e^{-\lambda(G(2 x)-G(z))} k(G(2 x)-G(z), 2 x) \frac{\psi(z)}{g(z)} d z .
$$

With (2.16), $\left(A_{g}\right)$ and lemma 3.1 this yields

$$
\left|e^{-\lambda(G(2 x)-G(z))} \cdot k(G(2 x)-G(z), 2 x) \cdot \frac{1}{g(z)}\right|<e^{-\left(R e \lambda+d_{\infty}\right)(G(2 x)-G(z)) \cdot} \frac{4}{g_{\min }}\|b\|_{\infty} \cdot e^{M} .
$$

Let $p=\operatorname{Re} \lambda+d_{\infty}$, then $p>0$, since $\lambda \in \Lambda$. Let the operator $K^{+}(p)$ be defined as

$$
\left(K^{+}(p) \psi\right)(x)=\int_{0}^{x\left(-a_{0}, 2 x\right)} e^{-p(G(2 x)-G(z))} \psi(z) d z .
$$


If we can prove that $K^{+}(p)$ is compact for all $p>0$ then it follows from Lemma 1 that $\hat{K}(\lambda)$ is compact for all $\lambda \in \Lambda$.

The following compactness criterium can be found in KUFNER et al. (1977).

LEMMA 2. The bounded linear operator $T: L_{1}(\Omega) \rightarrow L_{1}(\Omega)$ is compact if for every $\epsilon>0$ there exists a $\delta>0$ such that $\int_{\Omega}|(T \phi)(x+h)-(T \phi)(x)| d x<\epsilon\|\phi\|$ for all $\phi \in L_{1}(\Omega)$ and $|h|<\delta$.

We shall use this criterium to prove that $K^{+}(p)$ is compact for all $p>0$. For simplicity we assume that $g(x)=1$, for all $x$. The reader will have no difficulty to see that the proof can be carried through for more general $g$. Let $\psi \in L_{1}[0, \infty)$ and let $h>0$. Then

$$
\begin{aligned}
& \left|\left(K^{+}(p) \psi\right)(x+h)-\left(K^{+}(p) \psi\right)(x)\right|=\left|e^{-2 p(x+h)} \int_{0}^{2(x+h)-a_{0}} e^{p z} \psi(z) d z-e^{-2 p x} \int_{0}^{2 x-a_{0}} e^{p z} \psi(z) d z\right| \\
& \leqslant\left|e^{-2 p(x+h)}-e^{-2 p x}\right| \cdot \int_{0}^{2 x-a_{0}} e^{p z}|\psi(z)| d z+e^{-2 p(x+h)} \int_{2 x-a_{0}}^{2(x+h)-a_{0}} e^{p z}|\psi(z)| d z \stackrel{d e f}{=} f_{1}(x)+f_{2}(x),
\end{aligned}
$$

where $f_{1}(x)=\left(1-e^{-2 p h}\right)\left(K^{+}(p)|\psi|\right)(x), f_{2}(x)=e^{-2 p(x+h)} \int_{2 x-a_{0}}^{2(x+h)-a_{0}} e^{p z}|\psi(z)| d z$, and $|\psi|(x) \stackrel{d e f}{=}|\psi(x)|$. Thus

$$
\begin{aligned}
& \left\|f_{2}\right\|=\int_{0}^{\infty} f_{2}(x) d x=\int_{1 / a_{0}}^{\infty} e^{-2 p(x+h)} \cdot\left\{\int_{2 x-a_{0}}^{2(x+h)-a_{0}} e^{p z}|\psi(z)| d z\right\} d x \\
& =\int_{0}^{\infty} e^{p z}|\psi(z)| \cdot\left\{\int_{\frac{1}{2}\left(z+a_{0}\right)-h}^{\frac{1}{2}\left(z+a_{0}\right)} e^{-2 p(x+h)} d x\right\} d x=\frac{1-e^{-2 p h}}{2 p} e^{-p a_{0}}\|\psi\| .
\end{aligned}
$$

From these two estimates and Lemma 2, the compactness of $K^{+}(p)$ and thus $\hat{K}(\lambda)$ follows immediately. 